06,12

\title{
Низко- и инфранизкочастотная дисперсия диэлектрической проницаемости в матричном композите нанокристаллическая целлюлоза-триглицинсульфат
}

\author{
(C) Nguyen Hoai Thu'o'ng ${ }^{1}$, A.C. Сидоркин ${ }^{2,}$, С.Д. Миловидова ${ }^{2}$ \\ ${ }^{1}$ Industrial University of Ho Chi Minh City, \\ Ho Chi Minh City, Vietnam \\ ${ }^{2}$ Воронежский государственный университет, \\ Воронеж, Россия \\ I E-mail: sidorkin@phys.vsu.ru \\ (Поступила в Редакцию 4 сентября 2017 г. \\ В окончательной редакции 27 сентября 2017 г.)
}

Исследована дисперсия диэлектрической проницаемости композитов нанокристаллическая целлюлозатриглицинсульфат в диапазоне частот $10^{-3}-10^{6} \mathrm{~Hz}$ в области температур от комнатной до температуры фазового перехода данного композита $\left(54^{\circ} \mathrm{C}\right)$ в слабых электрических полях $\left(1 \mathrm{~V} \cdot \mathrm{cm}^{-1}\right)$. Показано, что в исследуемом диапазоне частот наблюдаются две области дисперсии: на инфранизких частотах $\left(10^{-3}-10 \mathrm{~Hz}\right)$ - диэлектрическая дисперсия, обусловленная миграционной поляризацией по механизму Максвелла-Вагнера, а в диапазоне более высоких частот $\left(10-10^{6} \mathrm{~Hz}\right)$ - дисперсия, обусловленная движением доменных стенок в кристаллитах триглицинсульфата в композите. Наличие остаточной воды в свежеприготовленных образцах приводит к появлению дополнительного низкотемпературного максимума в температурной зависимости диэлектрической проницаемости данного композита.

Исследование поддержано Российским научным фондом, проект № 17-72-20105.

DOI: 10.21883/FTT.2018.03.45561.256

\section{1. Введение}

Переход от микро- к наноэлектронике в существенной степени связан с развитием технологий получения новых материалов, в первую очередь, гетерогенных наносоставов и наноструктур, позволяющих значительно менять свойства используемых материалов в соответствии с потребностями практики на основе использования размерных эффектов на наноуровне. Среди указанных материалов особое место занимают композиты с сегнетоэлектриками в качестве внедренной компоненты, для которых за счет размягченности кристаллической решетки вблизи фазового перехода в сегнетоэлектрическое состояние диапазон вариации свойств гетерогенного материала оказывается особенно большим.

В таких материалах значительным изменениям, в первую очередь, подвергается сама температура фазового перехода в полярное состояние по сравнению с объемными сегнетоэлектриками [1-17]. В зависимости от материалов и размеров компонент сдвиг точки Кюри в композитных сегнетоэлектриках может достигать нескольких десятков градусов. При некоторых критических размерах внедряемых частиц в них вообще могут исчезать сегнетоэлектрические свойства [18]. Одновременно с этим в сегнетоэлектрических композитах меняются их диэлектрические и другие характеристики, в том числе спонтанная поляризация, коэрцитивное поле, доменная структура, диэлектрическая восприимчивость, включая зависимость диэлектрических свойств от частоты, и т.д.
Изучению диэлектрической дисперсии в сегнетоэлектрических композитах посвящено сравнительно немного исследований, хотя именно частотные зависимости диэлектрических свойств лежат в основе потенциальных применений данных материалов в электронике и радиочастотной технике. Настоящая работа представляет собой попытку частично восполнить указанный пробел.

До последнего времени для создания матричных сегнетоэлектрических композитов использовали в основном матрицы пористого оксида алюминия, кремния, стекла, опала [1-3,5-7,8-17]. В работах [5,19] нами впервые приведены результаты исследований диэлектрических свойств матричных композитов на основе нанокристаллической целлюлозы (NCC) в качестве матрицы и триглицинсульфата (TGS) в качестве сегнетоэлектрического включения. Работа [5] посвящена исследованию температурного поведения диэлектрических характеристик композита NCC+TGS в окрестности фазового перехода, а работа [19] - дисперсии диэлектрической проницаемости данного композита при комнатной температуре в диапазоне частот $10^{-3}-10^{3} \mathrm{~Hz}$. Для доказательного определения природы диэлектрической дисперсии данного нанокомпозита в настоящей работе исследование дисперсии диэлектрической проницаемости проводили в более широком диапазоне температур (от комнатной до температуры фазового перехода композита) и частот $\left(10^{-3}-10^{6} \mathrm{~Hz}\right)$. Кроме того, указанные исследования дополнены изучением проводимости и электрического модуля. 
Использование NCC в качестве матрицы для создания электрических композитов является относительно новым шагом [5]. Его привлекательность связана с тем, что в отличие от ранее использованных нанопористых матриц свойства нанокристаллической целлюлозы, как и свойства водородсодержащих сегнетоэлектриков, во многом определяются водородными связями. Поэтому исследование свойств нанокомпозита на основе NCC с водородсодержащим сегнетоэлектриком TGS представляет особенный интерес.

\section{2. Получение и структурные исследования образцов}

Для приготовления образцов композита NCC + TGS в качестве армирующей матрицы использовали гель-пленки нанокристаллической бактериальной целлюлозы Acetobacter Xylinum, структура которой представляет собой большое количество нанофибрил шириной 50-100 nm и длиной, превышающей диаметр в тысячу и более раз, сформированных гранями $(-110)$ и (110) [20-22]. В качестве сегнетоактивного наполнителя использовали соль триглицинсульфата $\left(\mathrm{NH}_{2} \mathrm{CH}_{2} \mathrm{COOH}\right)_{3} \cdot \mathrm{H}_{2} \mathrm{SO}_{4}$.

Для исследований диэлектрических свойств композита NCC + TGS использовали образцы из частично высушенной нанокристаллической целлюлозы с перпендикулярным расположением наноканалов относительно поверхности образца, приготовленные путем пятикратного удаления воды с двух поверхностей исходной нанокристаллической целлюлозы с помощью фильтровальной бумаги до уменьшения толщины гель-пленок примерно в 2 раза. На полученные частично подсушенные гель-пленки каплями в несколько этапов наносили насыщенный при комнатной температуре раствор TGS, подогретый до $50^{\circ} \mathrm{C}$, каждый раз до полного впитывания с двух сторон.

Подготовленные таким образом образцы выдерживали $3 \mathrm{~h}$ при температуре $100^{\circ} \mathrm{C}$ для полного удаления оставшейся воды и затем полностью высушивали при комнатной температуре. Из полученных пленок композитов толщиной $\sim 0.3-0.4 \mathrm{~mm}$ вырезали образцы площадью $\sim 60 \mathrm{~mm}^{2}$. На подготовленные образцы с помощью проводящего клея наносили электроды из сусального серебра.

Для получения информации о структуре приготовленных образцов использовали данные рентгеновской дифрактометрии, полученные с использованием для расшифровки экспериментальных дифрактограмм базы данных ICDD. Результаты рентгеновских исследований для матрицы нанокристаллической целлюлозы, монокристалла TGS и нанокомпозитов NCC+TGS представлены на рис. 1. Дифрактограммы для полностью высушенных образцов NCC (рис. 1, $a$ ) показали наличие линий при углах отражения $\sim 15^{\circ}$ и $23^{\circ}$, которые соответственно являются $[20,22]$ отражениями от плоскостей (-110) и (200) моноклинной решетки целлю-


Рис. 1. Дифрактограммы для матрицы нанокристаллической целлюлозы $\mathrm{NCC}(a)$, монокристалла TGS $(b)$ и нанокомпозитов $\mathrm{NCC}+\mathrm{TGS}(c)$.

лозы $I_{\beta}$ с межплоскостными расстояниями $d=0.610$ и $d=0.389-0.392 \mathrm{~nm}$.

На дифрактограмме композита NCC + TGS (рис. 1,c) присутствуют характерные линии как TGS, так и NCC.

\section{3. Экспериментальные результаты диэлектрических исследований и их обсуждение}

Диэлектрические измерения проводили с помощью цифрового моста LCR-meter 821, исследования дисперсии диэлектрической проницаемости - с помощью анализатора импеданса „Solartron-1260“ с диэлектрическим интерфейсом „Dielectric Interface-1296“ в слабом электрическом поле амплитудой $1 \mathrm{~V} \cdot \mathrm{cm}^{-1}$ в диапазоне частот $10^{-3}-10^{6} \mathrm{~Hz}$ при различных температурах от комнатной до температуры фазового перехода в 

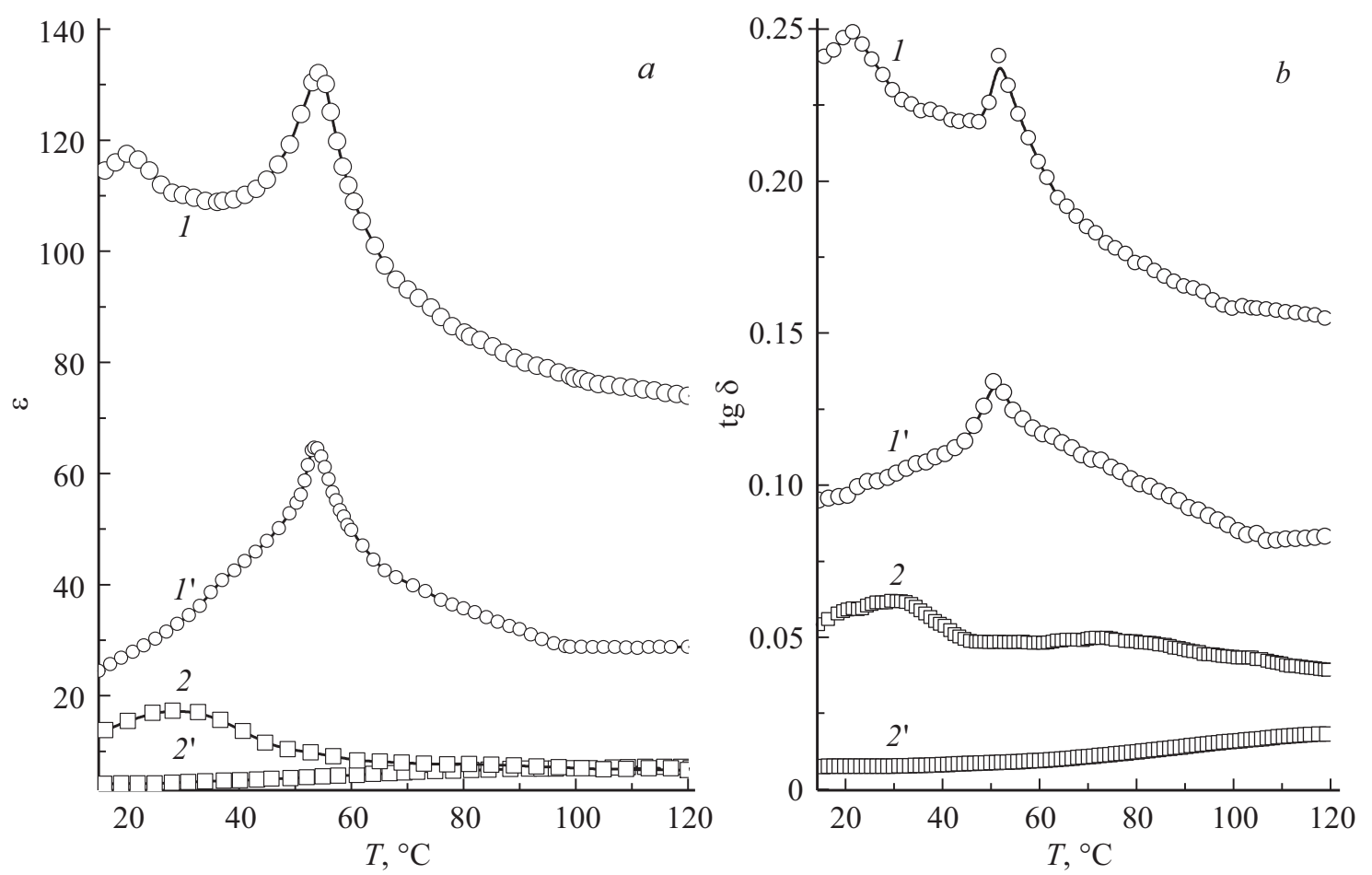

Рис. 2. Температурные зависимости диэлектрической проницаемости $(a)$ и тангенса диэлектрических потерь $(b)$ для нанокомпозитов NCC + TGS $\left(1,1^{\prime}\right)$ и матрицы нанокристаллической целлюлозы NCC $\left(2,2^{\prime}\right)$ до и после термической обработки.

данном композите $\left(54^{\circ} \mathrm{C}\right)$. Относительная погрешность измерений не превышала $0.1 \%$. Температуру измеряли цифровым термометром с точностью $0.1 \mathrm{~K}$.

3.1. Влияние остаточной воды на диэлектрические свойства. Из-за наличия системы водородных связей и в триглицинсульфате, и в матрице NCC за счет расположения на стенках наноканалов активных первичных групп ОН композиты NCC + TGS в свежеприготовленных образцах могут удерживать большое количество воды при получении композитов из водного раствора. В связи с этим представляет интерес исследование влияния остаточной воды на свойства композитов $\mathrm{NCC}+\mathrm{TGS}$.

На рис. 2 приведены температурные зависимости диэлектрической проницаемости $\varepsilon(T)$ (рис. $2, a)$ и тангенса угла диэлектрических потерь $\operatorname{tg} \delta(T)$ (рис. 2,b) для композитов NCC + TGS (кривые 1 и $1^{\prime}$ ), а также для матрицы NCC (кривые 2 и $2^{\prime}$ ) до и после отжига образцов. Для свежеприготовленных образцов композита NCC + TGS в зависимостях $\varepsilon(T)$ наблюдается два максимума: низкотемпературный при $\sim 20^{\circ} \mathrm{C}$ и высокотемпературный при $\sim 54^{\circ} \mathrm{C}$. После отжига низкотемпературный максимум исчезает. При этом как диэлектрическая проницаемость, так и тангенс диэлектрических потерь в композитах значительно снижаются во всем исследуемом температурном интервале. Температура высокотемпературного максимума практически не меняется после термической обработки образцов. Для исходной матрицы NCC низкотемпературные максимумы $\varepsilon(T)$ и $\operatorname{tg} \delta(T)$ проявляются до отжига при $\sim 30^{\circ} \mathrm{C}$, они сильно размыты и также исчезают после отжига образцов. Значения $\varepsilon$ и $\operatorname{tg} \delta$ для матрицы NCC малы и мало изменяются с температурой по сравнению с композитами NCC + TGS (кривые 2 и $2^{\prime}$, рис. 2).

Значительное снижение диэлектрической проницаемости и тангенса угла диэлектрических потерь, исчезновение низкотемпературных максимумов $\varepsilon(T)$ и $\operatorname{tg} \delta(f)$ в композитах NCC + TGS и в матрице NCC после термической обработки, очевидно, обусловлено испарением остаточной воды из свежеприготовленных образцов. В пользу высказанной гипотезы говорят и результаты аналогичных исследований для пленок желатина с триглицинсульфатом [23], где абсолютные значения диэлектрических характеристик значительно увеличиваются после выдержки образцов во влажной среде, а также наличие аналогичного рассматриваемому максимума диэлектрической проницаемости в полимеpax в присутствии связанных молекул воды [24].

Высокотемпературный максимум $\left(\sim 54^{\circ} \mathrm{C}\right)$, присутствующий в композитах NCC + TGS до и после отжига образцов и отсутствующий в матрице NCC до и после отжига, очевидно, может быть интерпретирован как максимум, соответствующий фазовому переходу в композите NCC + TGS, смещенному в область более высоких температур примерно на $5^{\circ} \mathrm{C}$ относительно точки Кюри объемного триглицинсульфата $\left(T_{c}=49^{\circ} \mathrm{C}\right)$. Как видно из рис. 2, $a$, указанный максимум сильно размыт, а значения диэлектрической проницаемости в области фазового переходе малы по сравнению с монокристаллом TGS, а также с композитами на основе других пористых матриц $[12,13,16]$ с включениями TGS. 

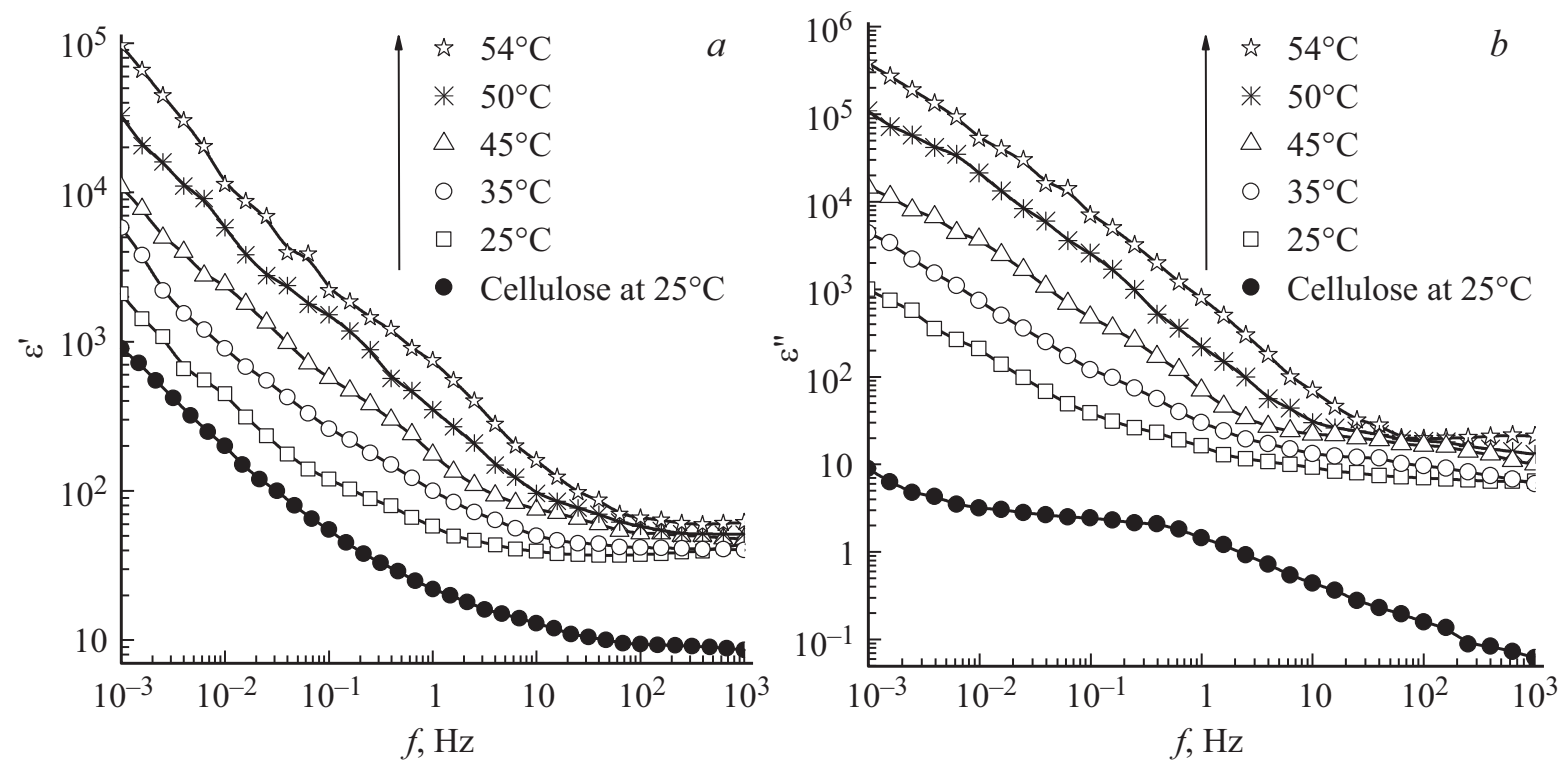

Рис. 3. Частотные зависимости действительной $(a)$ и мнимой $(b)$ частей диэлектрической проницаемости для нанокомпозитов NCC + TGS при различных температурах и для матрицы нанокристаллической целлюлозы NCC при комнатной температуре.

3.2. Дисперсия диэлектрической проницае мости. Исследования дисперсии диэлектрической проницаемости для образцов композита NCC + TGS и матрицы NCC проводили после термической обработки. Частотные зависимости действительной $\varepsilon^{\prime}(f)$ и мнимой $\varepsilon^{\prime \prime}(f)$ частей диэлектрической проницаемости в диапазоне инфранизких и низких частот $\left(10^{-3}-10^{3} \mathrm{~Hz}\right)$ при различных температурах от комнатной до температуры фазового перехода в композите NCC + TGS, a также для матрицы NCC при комнатной температуре представлены на рис. 3 в двойном логарифмическом масштабе.

Как видно из рис. 3, в указанном диапазоне частот зависимости как $\varepsilon^{\prime}(f)$ (рис. $\left.3, a\right)$, так и $\varepsilon^{\prime \prime}(f)$ (рис. $\left.3, b\right)$ имеют две области дисперсии при всех исследуемых температурах: в инфрачастотной области $\left(10^{-3}-10^{1} \mathrm{~Hz}\right)$ значения действительной $\varepsilon^{\prime}(f)$ и мнимой $\varepsilon^{\prime \prime}(f)$ частей диэлектрической проницаемости уменьшаются с ростом частоты по универсальному степенному закону релаксации $\left(\varepsilon^{\prime}, \varepsilon^{\prime \prime \propto} f^{n}\right)[25,26]$. В более высокочастотной области $\left(10^{1}-10^{3} \mathrm{~Hz}\right)$ наблюдается незначительное

Показатель степенного закона в зависимостях действительной и мнимой частей от температуры на низко- и инфранизких частотах для композита NCC + TGS

\begin{tabular}{c|c|c}
\hline \multirow{2}{*}{$T,{ }^{\circ} \mathrm{C}$} & \multicolumn{2}{|c}{$n$} \\
\cline { 2 - 3 } & $\varepsilon^{\prime}$ & $\varepsilon^{\prime \prime}$ \\
\hline 25 & 0.67 & 0.73 \\
35 & 0.58 & 0.79 \\
45 & 0.65 & 0.89 \\
50 & 0.75 & 0.91 \\
54 & 0.80 & 0.87
\end{tabular}

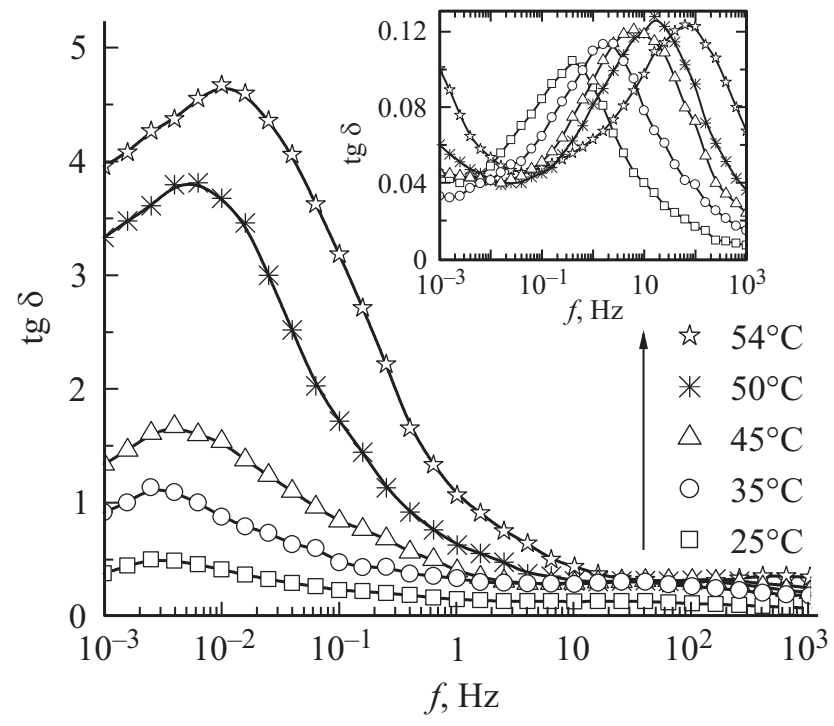

Рис. 4. Частотные зависимости тангенса диэлектрических потерь для нанокомпозитов NCC + TGS при различных температурах, на вставке - для матрицы нанокристаллической целлюлозы NCC.

уменьшение $\varepsilon^{\prime}(f)$ и $\varepsilon^{\prime \prime}(f)$ с ростом частоты измерительного поля. Рассчитанные (с использованием вышеприведенных зависимостей компонент диэлектрической проницаемости от частоты) близкие к единице значения $n$ для композита NCC + TGS при исследуемых температурах (см. таблицу) подтверждают значительную зависимость $\varepsilon^{\prime}$ и $\varepsilon^{\prime \prime}$ от частоты в инфранизкочастотной области.

Частотные зависимости тангенса угла диэлектрических потерь $\operatorname{tg} \delta(f)$ (рис. 4) в инфранизкочастотной 


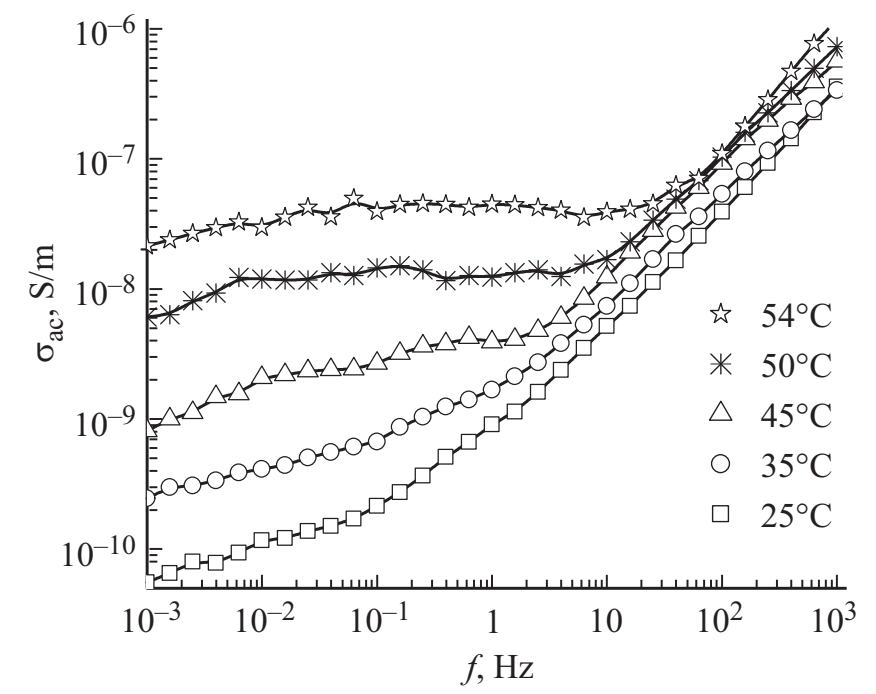

Рис. 5. Частотные зависимости проводимости для нанокомпозитов NCC + TGS при различных температурах.

области демонстрируют наличие максимумов, которые становятся более выраженными при повышении температуры. С ростом температуры увеличиваются и значения $\varepsilon^{\prime}$ и $\varepsilon^{\prime \prime}$ (рис. 4); $\varepsilon^{\prime}, \varepsilon^{\prime \prime}$ и $\operatorname{tg} \delta$ для композита $\mathrm{NCC}+$ TGS в инфранизкочастотной области оказываются значительно бо́льшими, чем для монокристалла TGS при соответствующих исследуемых температурах. Например, при фазовом переходе на частоте $10^{-3} \mathrm{~Hz}$ для монокристалла TGS $\varepsilon^{\prime} \approx 10^{4}$ [27], а для композита $\mathrm{NCC}+\mathrm{TGS} \varepsilon^{\prime} \approx 10^{6}$. Наличие максимумов тангенса угла диэлектрических потерь $\operatorname{tg} \delta(f)$ наблюдается и для матрицы NCC при всех исследуемых температурах (на вставке к рис. 4). При этом $\operatorname{tg} \delta, \varepsilon^{\prime}$ и $\varepsilon^{\prime \prime}$ для матрицы малы по сравнению с композитом NCC + TGS.

Указанные частотные зависимости $\varepsilon^{\prime}(f), \varepsilon^{\prime \prime}(f)$, изменяющиеся по универсальному степенному закону ре- лаксации, а также наличие максимумов тангенса угла диэлектрических потерь $\operatorname{tg} \delta(f)$ в инфранизкочастотном диапазоне обусловлено, по-видимому, наличием проводимости в композите. На это указывает частотное поведение эффективной проводимости, рассчитанной на основании диэлектрических потерь по формуле:

$$
\sigma_{a c}=\omega \varepsilon_{0} \varepsilon^{\prime \prime},
$$

где $\omega=2 \pi f-$ круговая частота измерительного поля, $\varepsilon_{0}$ - диэлектрическая постоянная вакуума, $\varepsilon^{\prime \prime}-$ мнимая часть комплексной диэлектрической проницаемости.

Полученные на основании уравнения (1) результаты расчетов представлены на рис. 5. Как видно из рис. 5, чем больше температура, тем более выраженными становятся пологие, независящие от частоты участки зависимости эффективной проводимости, которые расширяются в сторону более высоких частот при повышении температуры. Помимо этого, значения проводимости на низких частотах значительно увеличиваются с ростом температуры.

Наличие пологих, не зависящих от частоты участков $\sigma_{a c}$, соответствующих статической проводимости, обычно связано с приэлектродной поляризацией, обусловленной накоплением зарядов на границе композитэлектрод $[28,29]$. Для исключения влияния указанных эффектов на релаксацию проводимости композита $\mathrm{NCC}+$ TGS удобно использовать электрический модуль, который определяется в виде обратной комплексной диэлектрической проницаемости [29,30]:

$$
M^{*}=\frac{1}{\varepsilon^{*}}=M^{\prime}+i M^{\prime \prime}=\frac{\varepsilon^{\prime}}{\varepsilon^{\prime 2}+\varepsilon^{\prime \prime 2}}+i \frac{\varepsilon^{\prime \prime}}{\varepsilon^{\prime 2}+\varepsilon^{\prime 2}} .
$$

Полученные с помощью уравнения (2) частотные зависимости действительной $M^{\prime}(f)$ и мнимой $M^{\prime \prime}(f)$ частей электрического модуля для композита $\mathrm{NCC}+\mathrm{TGS}$ приведены на рис. 6. Как видно из рис. 6, поведение
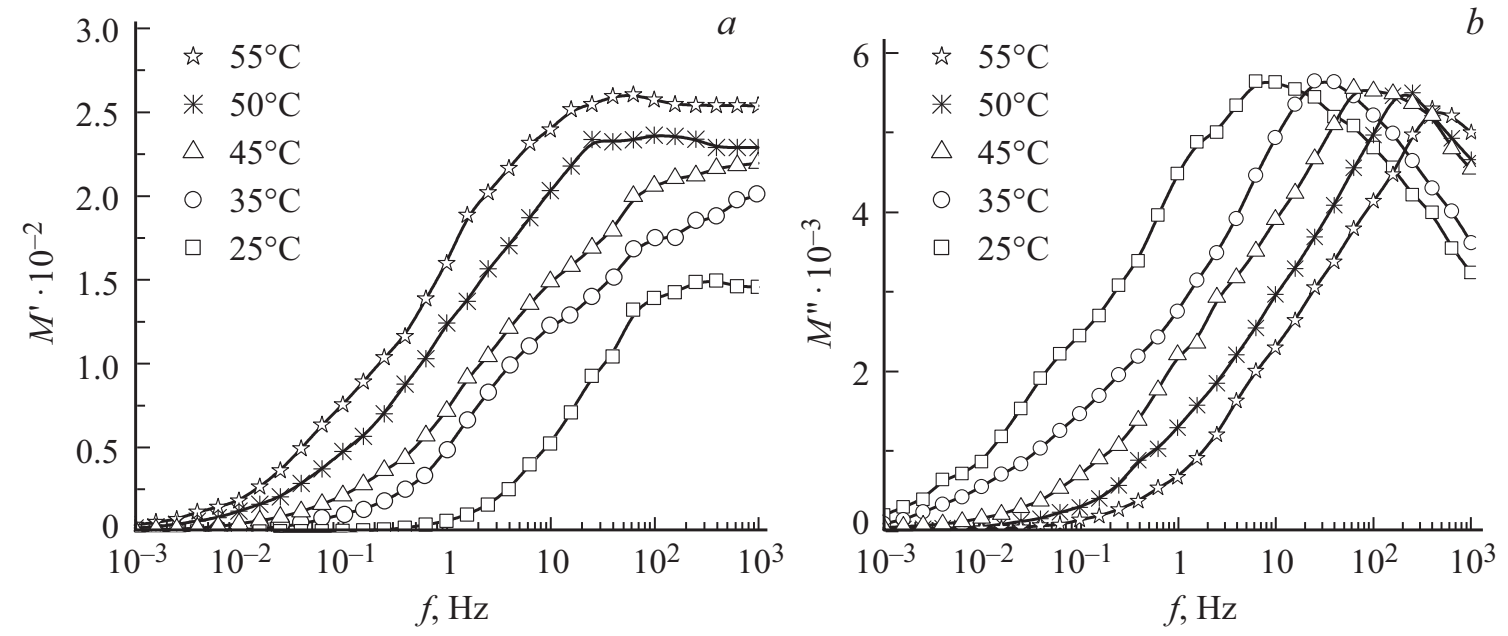

Рис. 6. Частотные зависимости действительной $(a)$ и мнимой $(u)$ частей электрического модуля для нанокомпозитов NCC + TGS при различных температурах. 

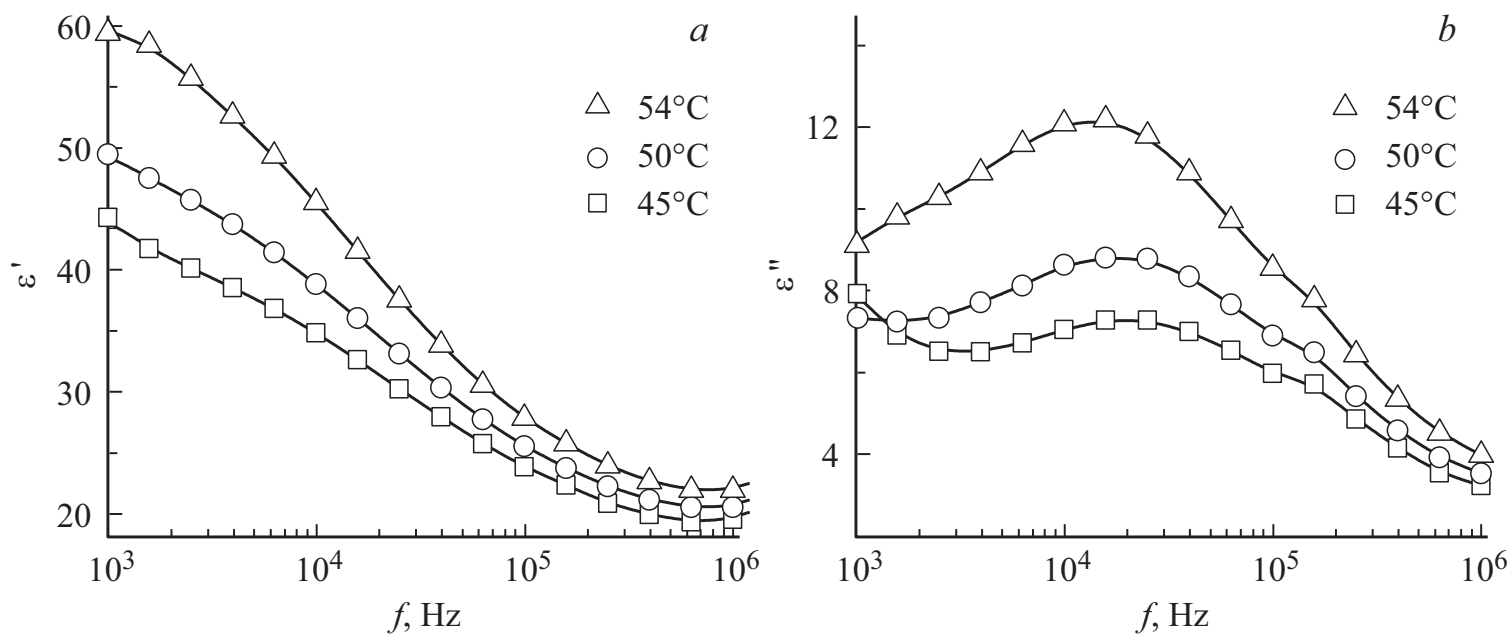

Рис. 7. Частотные зависимости действительной $(a)$ и мнимой $(b)$ частей диэлектрической проницаемости для нанокомпозитов NCC + TGS при различных температурах.

$M^{\prime}(f)$ и $M^{\prime \prime}(f)$ в исследуемых материалах характеризуется типичным для процессов релаксации проводимости поведением: действительная часть электрического модуля $M^{\prime}$ возрастает до насыщения с ростом частоты при наличии максимума $M^{\prime \prime}(f)$, который смещается в область более высоких частот с ростом температуры. Указанное поведение для $M^{\prime}(f)$ и $M^{\prime \prime}(f)$ наблюдается также для матрицы NCC в данном диапазоне частот.

Зависимости времени релаксации $\tau_{M}$ от температуры $\left(\tau_{M}=1 / 2 \pi f\right.$, где $f$ - частота релаксации, соответству-
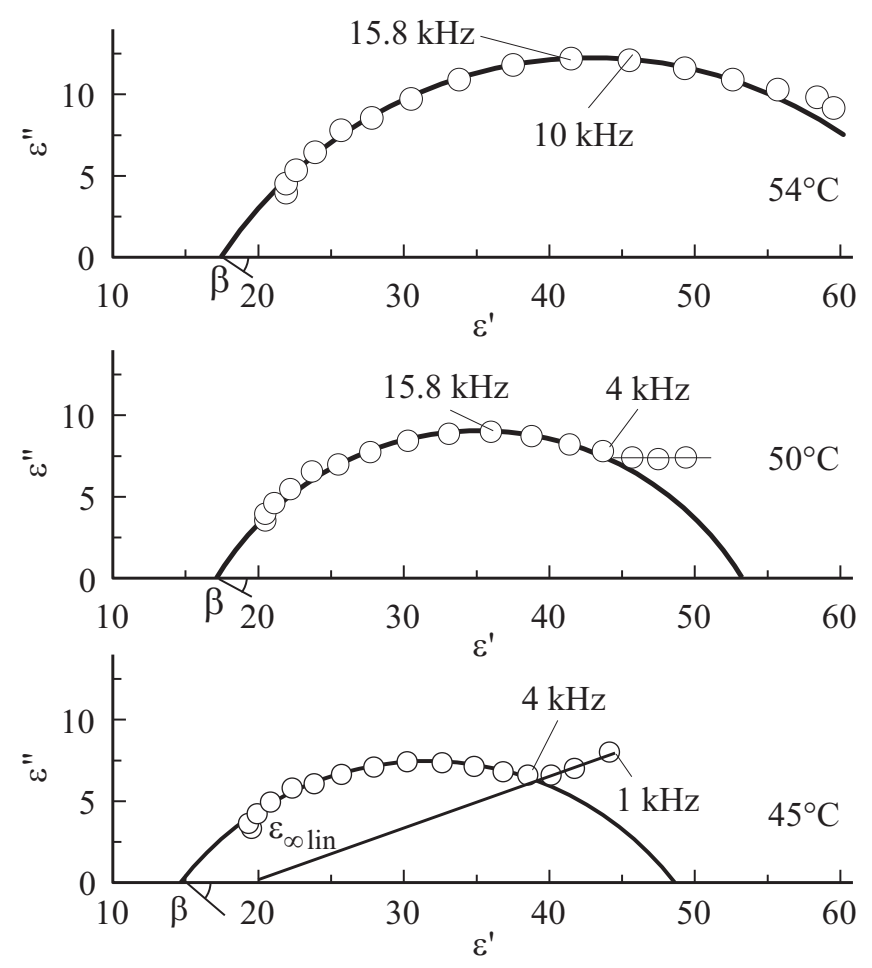

Pис. 8. Диаграммы Коула-Коула для нанокомпозитов NCC + TGS при различных температурах. ющая максимумам $M^{\prime \prime}(f)$ для композита NCC + TGS и $\mathrm{NCC}$, показали хорошее согласие с законом Аррениуса. Полученные энергии активации составляют $0.96 \mathrm{eV}$ для композита NCC + TGS и $0.52 \mathrm{eV}$ для матрицы NCC.

На рис. 7 представлены результаты исследований частотных зависимостей действительной $\varepsilon^{\prime}(f)$ (рис. 7, $\left.a\right)$ и мнимой $\varepsilon^{\prime \prime}(f)$ (рис. 7,b) частей комплексной диэлектрической проницаемости для композита $\mathrm{NCC}+\mathrm{TGS}$ в диапазоне частот $10^{3}-10^{6} \mathrm{~Hz}$ при различных температурах, а на рис. 8 - соответствующие им диаграммы Коула-Коула.

Как следует из рис. 7, с ростом температуры от комнатной до температуры фазового перехода в композите $\mathrm{NCC}+\mathrm{TGS}$ на частотной зависимости мнимой $\varepsilon^{\prime \prime}(f)$ (рис. $7, b)$ части комплексной диэлектрической проницаемости наблюдается появление максимума, который лежит в области более низких частот $\left(10^{4}-10^{5} \mathrm{~Hz}\right)$ по сравнению с монокристаллом TGS $\left(10^{5}-10^{6} \mathrm{~Hz}\right)$ [31-36]. При этом присутствующий при более низких температурах на диаграмме Коула-Коула участок линейной дисперсии с ростом температуры становится менее выраженным и полностью исчезает при фазовом переходе $\left(54^{\circ} \mathrm{C}\right)$.

\section{4. Заключение}

Таким образом, проведенные исследования показали значительное влияние матрицы на свойства композитов $\mathrm{NCC}+$ TGS. По нашему мнению, фиксация поляризации в сегнетоэлектрическом включении водородными связями с матрицей приводит к смещению температуры фазового перехода в данном композите в область более высоких температур. Размытие фазового перехода, по всей видимости, обусловлено разбросом диаметров наноканалов в исходной матрице NCC и неодинаковым их заполнением нанокристаллами TGS.

В диапазоне инфранизких частот $\left(10^{-3}-10 \mathrm{~Hz}\right)$ дисперсию диэлектрической проницаемости в композите 
определяют процессы релаксации миграционной поляризации по механизму Максвелла-Вагнера, где в роли носителей заряда могут выступать заряженные примеси, содержащиеся в присутствующей в композите воде.

В диапазоне частот $10-10^{6} \mathrm{~Hz}$ в композите NCC+TGS наблюдаются характерная для триглицинсульфата релаксация дебаевского типа и дисперсия линейного типа, обусловленные соответственно обратимым и необратимым движением доменных стенок в кристаллитах триглицинсульфата в композите.

Выявленные причины изменения диэлектрических свойств исследуемых нанокомпозитов могут рассматриваться в качестве достаточно эффективных способов управления практически полезными свойствами сегнетоэлектрических композитов.

\section{Список литературы}

[1] M. Neeraj, D.N. Arvind, S.H. Jasbir, G.D. Varma, N.P. Pathak, R. Nath. Ferrelectrics Lett. 42, 75 (2015).

[2] M. Pritula, A.V. Kosinova, M.I. Kolybaeva, O.N. Bezkrovnaya, V.V. Grebenev, A.E. Voloshin, D.A. Vorontsov, D.S. Sofronov, O.M. Vovk, V.N. Baumer. Cryst. Res. Technol. 49, 345 (2014).

[3] A. Cizman, M. Tomasz, E. Dirk, B. Andrei, P. Ryszard. J. Nanopart. Res. 15, 1756 (2013).

[4] С.В. Барышников, Е.В. Чарная, А.Ю. Милинский, Ю.В. Патрушев. ФТТ 55, 2439 (2013).

[5] S.D. Milovidova, O.V. Rogazinskaya, A.S. Sidorkin, H.T. Nguyen, E.V. Grohotova. Ferroelectrics 469, 116 (2014).

[6] L. Korotkov, V. Dvornikov, M. Vlasenko, T. Korotkova, A. Naberezhnov, E. Rysiakiewicz-Pasek. Ferroelectrics 444, 100 (2013).

[7] M.J. Uddin, T.R. Middya, N.G. Popravko. Curr. Appl. Phys. 13, 461 (2013).

[8] S.V. Baryshnikov, A.Yu. Milinskiy, E.V. Charnaya, A.S. Bugaev, M.I. Samoylovich. Ferroelectrics 493, 85 (2016).

[9] L. Singh, B. Kaur, N. Kumar, D.Y. Jeong, N. Dabra, J.S. Hundal. Int. J. Electrochem. Sci. 11, 4037 (2016).

[10] А.Г. Горчаков, П.С. Седых, Е.В. Чарная, С.В. Барышников, C. Tien, D. Michel. ФTT 51, 2028 (2009).

[11] C. Tien, E.V. Charnaya, M.K. Lee, S.V. Baryshnikov, D. Michel, W. Bohlman. J. Phys.: Condens. Matter 20, 215205 (2008).

[12] O.V. Rogazinskaya, S.D. Milovidova, A.S. Sidorkin, N.G. Popravko, M.A. Bosykh, V.S. Enshina. Ferroelectrics 397, 191 (2010).

[13] A. Cizman, T. Antropova, I. Anfimova, I. Drozdova, E. Rysiakiewicz-Pasek, E.B. Radojewska, R. Poprawski. J. Nanopart. Res. 15, 1087 (2013).

[14] С.В. Барышников, Е.В. Чарная, А.Ю. Милинский, Ю.В. Патрушев, А.Ю. Гойхман, С. Tien, M.K. Lee, L.J. Chang. ФTT 55, 987 (2013).

[15] S.V. Baryshnikov, C. Tien, E.V. Charnaya, M.K. Lee, D. Michel, W. Böhlmann, E.V. Stukova. Ferroelectrics 363, 177 (2008).

[16] N.G. Popravko, A.S. Sidorkin, S.D. Milovidova, O.V. Rogazinskaya. Ferroelectrics 443, 8 (2013).

[17] А.Л. Пирозерский, Е.В. Чарная, С. Tien. ФТТ 49, 327 (2007).

[18] В.М. Фридкин. УФН 176, 203 (2006).
[19] Х.Т. Нгуен, С.Д. Миловидова, А.С. Сидоркин, О.В. Рогазинская. ФТТ 57, 491 (2015).

[20] Yu.G. Baklagina, A.K. Khripunov, A.A. Tkachenko, S.V. Gladchenko, V.K. Lavrentev, A.Ya. Volkov, V.K. Nilova, V.M. Denisov, T.E. Sukhanova, I.S. Zanaveskina, V.V. Klechkovskaya, L.A. Feigin. J. Appl. Chem. 76, 989 (2003).

[21] A.K. Khripunov, A.A. Tkachenko, Yu.G. Baklagina, L.N. Borovikiva, V.K. Nilova, R.Yu. Smyslov, V.V. Klechkovskaya, N.A. Matveeva, A.Ya. Volkov, V.K. Lavrentev, M.E. Vylegzhanina, T.E. Sukhanova, V. Kopeikin. J. Appl. Chem. 80, 1549 (2007).

[22] Yu.G. Baklagina, A.K. Khripunov, A.A. Tkachenko, V.V. Kopeikin, N.A. Matveeva, V.K. Lavrentev, V.K. Nilova, T.E. Sukhanova, R.Yu. Smyslov, I.S. Zanaveskina, V.V. Klechkovskaya, L.A. Feigin. J. Appl. Chem. 78, 1176 (2005).

[23] V.E. Khutorsky, S.B. Lang. J. Appl. Phys. 82, 1288 (1997).

[24] Г.В. Маркин, И.А. Малышкина, Н.Д. Гаврилова, Е.Е. Махаева, Т.Е. Григорьев. Вестн. МГУ. Сер. 3. Физика, астрономия 6,42 (2008).

[25] A.K. Jonscher. Nature (London) 267, 673 (1977).

[26] A.K. Jonscher. Dielectric Relaxation in Solids. Chelsea Dielectric. Press Limited, London (1983). 380 p.

[27] А.М. Лотонов, В.К. Новик, Н.Д. Гаврилова. Вестн. МГУ. Cер. 3. Физика, астрономия 5, 27 (2006).

[28] M.M. Gomaa, P. Alikaj. Mar. Geophys. Res. 30, 265 (2010).

[29] H. Lu, X. Zhang, H. Zhang. J. Appl. Phys. 100, 054104 (2006).

[30] K. Pathmanathan, G.P. Johari. J. Chem. Phys. 95, 5990 (1991).

[31] А.В. Шильников, Е.В. Галиярова, Д.Г. Васильев, С.В. Горин, Л.А. Шувалов. Изв. РАН. Сер. физ. 60, 193 (1996).

[32] Н.М. Гаврилова. ФТТ 31, 248 (1989).

[33] N.M. Galiyarova. Ferroelectrics 170, 111 (1995).

[34] V. Alexandru, C. Mindru, C. Berbecaru. Dig. J. Nanomater. Biostruct. 7, 1353 (2012).

[35] C. Mindru, C.P. Ganea, H.V. Alexandru. J. Optoelectron. Adv. Mater. 14, 157 (2012).

[36] J. Zhang. Ferroelectrics 281, 105 (2002). 